\title{
Multilayer Active Shell Mirrors for Space Telescopes
}

\author{
John Steeves ${ }^{a}$, Kathryn Jackson ${ }^{b}$, Sergio Pellegrino ${ }^{b}$, David Redding ${ }^{a}$, J. Kent Wallace $^{a}$, \\ Samuel Case Bradford ${ }^{a}$, Troy Barbee ${ }^{c}$ \\ ${ }^{a}$ Jet Propulsion Laboratory, California Institute of Technology, 4800 Oak Grove Dr, \\ Pasadena, CA 91109; \\ ${ }^{b}$ California Institute of Technology, 1200 E. California Blvd, Pasadena, CA 91125; \\ ${ }^{c}$ Lawrence Livermore National Laboratory, 7000 East Ave, Livermore, CA 94550
}

\begin{abstract}
A novel active mirror technology based on carbon fiber reinforced polymer (CFRP) substrates and replication techniques has been developed. Multiple additional layers are implemented into the design serving various functions. Nanolaminate metal films are used to provide a high quality reflective front surface. A backing layer of thin active material is implemented to provide the surface-parallel actuation scheme. Printed electronics are used to create a custom electrode pattern and flexible routing layer. Mirrors of this design are thin $(<1.0 \mathrm{~mm})$, lightweight $\left(2.7 \mathrm{~kg} / \mathrm{m}^{2}\right)$, and have large actuation capabilities. These capabilities, along with the associated manufacturing processes, represent a significant change in design compared to traditional optics. Such mirrors could be used as lightweight primaries for small CubeSat-based telescopes or as meter-class segments for future large aperture observatories. Multiple mirrors can be produced under identical conditions enabling a substantial reduction in manufacturing cost and complexity.

An overview of the mirror design and manufacturing processes is presented. Predictions on the actuation performance have been made through finite element simulations demonstrating correctabilities on the order of $250-300 \times$ for astigmatic modes with only 41 independent actuators. A description of the custom metrology system used to characterize the active mirrors is also presented. The system is based on a Reverse Hartmann test and can accommodate extremely large deviations in mirror figure ( $>100 \mu \mathrm{m} \mathrm{PV})$ down to sub-micron precision. The system has been validated against several traditional techniques including photogrammetry and interferometry. The mirror performance has been characterized using this system, as well as closed-loop figure correction experiments on $150 \mathrm{~mm}$ dia. prototypes. The mirrors have demonstrated post-correction figure accuracies of $200 \mathrm{~nm}$ RMS (two dead actuators limiting performance).
\end{abstract}

Keywords: Active Mirrors, CFRP, Nanolaminate, Replication, Reverse Hartmann

\section{INTRODUCTION \& BACKGROUND}

Several large-aperture space-based mission concepts are currently under study for the 2020 Decadal Survey. Perhaps the largest and most complex is the Large UV/Optical/IR Surveyor (LUVOIR) with a 10 - $12 \mathrm{~m}$ class primary aperture. ${ }^{1,2}$ At this scale, it is required to package and deploy the primary aperture using smaller mirror segments in order to fit within the payload fairing of current launch vehicles. Mirror segmentation is currently implemented with the James Webb Space Telescope (JWST), which uses 18 hexagonal mirrors to construct the $6.5 \mathrm{~m}$ dia. primary aperture. However, with aspheric apertures, identical segments cannot be used as varying prescriptions are required depending on the segment position with respect to the optical axis. For JWST 3 unique segments are required, however this number grows rapidly as the diameter of the primary mirror increases. This is a current technological challenge experienced by large ground-based telescopes. For example, the Thirty Meter Telescope (TMT) implementing a $30 \mathrm{~m}$ dia. primary is composed of 492 total segments with 82 unique prescriptions. ${ }^{3}$ Therefore, a substantial manufacturing effort is required in order to construct an telescope of this size.

For further information contact John Steeves: john.b.steeves@jpl.nasa.gov

Advances in Optical and Mechanical Technologies for Telescopes and Instrumentation II, edited by Ramón Navarro, James H. Burge, Proc. of SPIE Vol. 9912, 99121K

(c) 2016 SPIE · CCC code: 0277-786X/16/\$18 - doi: 10.1117/12.2233594

Proc. of SPIE Vol. $991299121 \mathrm{~K}-1$ 
Existing mirror technologies that implement grinding and polishing techniques result in costly, complex, and timely efforts. Furthermore, the areal density of such mirrors is prohibitively high. A method of producing lightweight mirrors while reducing manufacturing complexity is to implement replication techniques. In this process a convex mandrel is used to define the optical figure. The mirror material is then placed upon this surface and a curing process is initiated, often under the application of high temperature and pressure. Once the cure is complete and the substrate is removed from the mandrel, a concave mirror is produced. Multiple segments can be produced from a single mandrel, resulting in a high through-put manufacturing process. A significant amount of work has been performed in an attempt to produce mirrors using replication techniques. ${ }^{4-8}$ However, these technologies often suffer from a lack of surface accuracy as internal stresses developed during the replication process tend to cause distortions.

Advanced mirror technologies, such as those implementing distributed actuation, can help to overcome the above challenges. Active mirrors not only allow for corrections of initial manufacturing errors, but can also provide in-situ control of figure deformations resulting from thermal variations, gravity sag, and/or material creep. This scheme can significantly reduce the cost and complexity associated with segment fabrication as well as system I\&T as diffraction limited performance can be demonstrated under a variety of test conditions (i.e. room/cryogenic temperatures, zero/1g). Furthermore, with large actuation strokes, bulk changes to the optical prescription of each segment can be realized, potentially enabling 1) identical segments to be used in a large aperture telescope and/or 2) reconfiguration of the aperture geometry in order to change the resolution of the telescope as whole. ${ }^{9,10}$

This paper presents a novel active mirror technology based on carbon fiber reinforced polymer (CFRP) substrates and replication techniques. Additional material layers are also implemented into the design serving various functions. An overview of the multilayer active mirror concept is shown in Figure 1. A CFRP substrate is used to provide the overall shape and structure of the mirror. A reflective nanolaminate facesheet is then bonded to the front of the substrate providing a high-quality reflective surface. A layer of active material, with electrodes on its top and bottom surfaces, is incorporated onto the back of the CFRP substrate providing the surface-parallel actuation scheme. Finally, a flexible electrode routing layer is used to access each electrode. The entire mirror is kept extremely thin $(<1.0 \mathrm{~mm})$ and thus lightweight $\left(\sim 2.5 \mathrm{~kg} / \mathrm{m}^{2}\right)$. The mirror is free-standing with no backing structure for support, allowing for large actuation strokes. Surface replication techniques along with bonding and transfer processes are implemented, eliminating the need for grinding/polishing steps. Mirrors of this design could be used as lightweight primaries for small CubeSatbased telescopes or as meter-class segments for future large aperture observatories. Multiple mirrors can be produced under identical conditions which produces a substantial reduction in manufacturing cost and complexity.

The paper is structured as follows: Section 2 gives an overview of the components that make up the multilayer mirror technology. Section 3 provides figure correction predictions, including correctability and stroke over independent Zernike modes, made through finite element simulations. An overview of the Reverse Hartmann metrology system is provided in Section 4. Section 5 details the performance of a $150 \mathrm{~mm}$ diameter active mirror prototype, and finally Section 6 summarizes and concludes the paper.

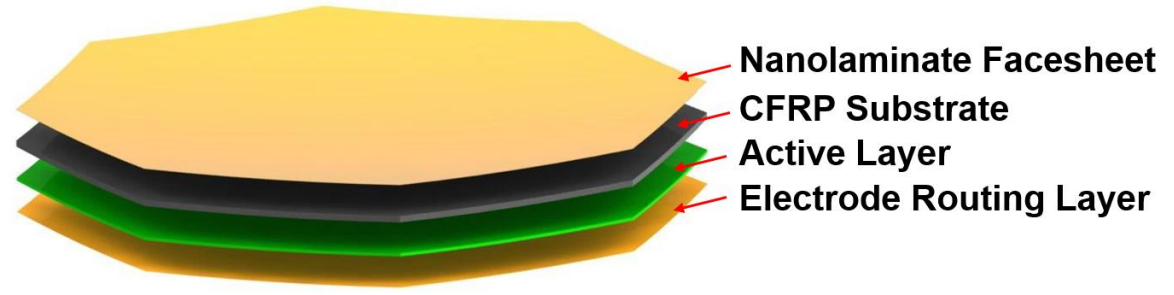

Figure 1. Exploded view of the multilayer active mirror technology. 


\section{MIRROR COMPONENTS}

\subsection{CFRP Substrate}

The CFRP substrates are constructed from unidirectional material consisting of M55J fibers embedded in an epoxy matrix. The material is manufactured using tow-spreading techniques and can thus be made extremely thin. For this work, each ply is only $30 \mu \mathrm{m}$ thick which is approximately $4-5 \times$ thinner than conventional composite materials. Table 1 displays the material properties of this material, obtained experimentally. The individual plies are highly orthotropic as the modulus in the fiber direction, E1, is over 50 times greater than that in the transverse direction, E2. Therefore, to produce shells with more isotropic properties, multidirectional laminates were constructed. A study was performed in order to determine the optimal laminate orientation for the active mirror application. Table 3 lists the considered laminates for 8,16 , and 32 ply counts which are all a combination of plies oriented in the $0^{\circ}, 90^{\circ}$ or $\pm 45^{\circ}$ directions. However, the specific stacking sequence will determine the mechanical response of each laminate. Two variants of the 16 and 32-ply laminates were considered: $\mathrm{a}$ and $\mathrm{b}$. Laminate $2 \mathrm{a}$ is defined by stacking two layers of the 8-ply laminate on top of each other. Laminate $2 \mathrm{~b}$ differs from $2 \mathrm{a}$ in that the second 8 -ply layer is rotated $90^{\circ}$ with respect to the first. Laminates $3 \mathrm{a}$ and $3 \mathrm{~b}$ are constructed in the same manner; however, four layers are implemented.

Table 1. Material properties of M55J carbon fibers embedded in ThinPreg ${ }^{T M} 120 \mathrm{EPHTg}-1$ epoxy $\left(\mathrm{V}_{f} \approx 50 \%\right)$.

\begin{tabular}{lr}
\hline \hline Property & Value \\
\hline$E_{1}(\mathrm{GPa})$ & 340 \\
$E_{2}(\mathrm{GPa})$ & 6.0 \\
$G_{12}(\mathrm{GPa})$ & 4.2 \\
$\nu_{12}$ & 0.35 \\
\hline${ }^{*}$ Characterization courtesy of Yuchen Wei. &
\end{tabular}

Table 2. Considered CFRP laminate orientations

\begin{tabular}{ccc}
\hline \hline Laminate ID & Ply-Count & Laminate Orientation \\
\hline 1 & 8 & {$\left[0^{\circ} /+45^{\circ} /-45^{\circ} / 90^{\circ}\right]_{s}$} \\
$2 \mathrm{a}$ & 16 & {$\left[0^{\circ} /+45^{\circ} /-45^{\circ} / 90^{\circ}\right]_{2 s}$} \\
$2 \mathrm{~b}$ & 16 & {$\left[0^{\circ} /+45^{\circ} /-45^{\circ} / 90^{\circ}\right]_{s}+\left[90^{\circ} /-45^{\circ} /+45^{\circ} / 0^{\circ}\right]_{s}$} \\
$3 \mathrm{a}$ & 32 & {$\left[0^{\circ} /+45^{\circ} /-45^{\circ} / 90^{\circ}\right]_{4 s}$} \\
$3 \mathrm{~b}$ & 32 & {$\left[\left[0^{\circ} /+45^{\circ} /-45^{\circ} / 90^{\circ}\right]_{s}+\left[90^{\circ} /-45^{\circ} /+45^{\circ} / 0^{\circ}\right]_{s}\right]_{2}$}
\end{tabular}

Using Classical Lamination Theory (CLT) ${ }^{11}$ the in-plane stiffness, A, bending stiffness, D, and coupling, $\mathbf{B}$, matrices can be computed from the material properties of each ply, and the stacking sequence (see Jones, $\mathrm{R}(1998)^{11}$ for the formulation of the ABD matrix). For all of the considered laminate orientations, the $\mathbf{B}$ matrix is null and therefore there exists no coupling between in-plane loads and out-of-plane curvatures. The principal in-plane stiffness (A11) and bending stiffness (D11) are shown in Figure 2 at varying orientations with respect to the laminate coordinate system ( $\mathrm{CCW}$ with respect to the $0^{\circ}$ ply). Both of the stiffness components are normalized by their maximum values for each laminate. It can be seen that all laminates possess in-plane isotropy as A11 is independent of orientation. This result gives rise to the traditional classification of a "quasi-isotropic" laminate. However, for active mirror applications, the bending properties are equally important. From Figure 2 it is apparent that significant variations exist in the bending stiffness over the range of laminates considered. The magnitude and orientation of the maximum reduction in D11 is summarized in Table 3. The 8-ply laminate displays the greatest variation in bending stiffness, with a reduction factor of 0.186 orientated at $65.0^{\circ}$.

A more notable comparison is made between Laminates $2 \mathrm{a}$ and $2 \mathrm{~b}$. Although the laminates have the same number of plies, Laminate $2 \mathrm{~b}$ displays a higher degree of isotropy with respect to bending stiffness with a maximum reduction factor of only 0.972 in comparison to 0.687 . The same effect is observed when comparing 
the bending stiffness of Laminates 3a and 3b, where comparative reduction factors of 0.911 to 0.993 are observed. This increased level of isotropy is desired, as the response of the laminate is no longer dependent on orientation. Due to this result, laminates $2 \mathrm{~b}$ and $3 \mathrm{~b}$ were implemented and are referred as the "16-ply" and "32-ply" laminates for simplicity. This result also demonstrates the utility of using thin-ply material. 16 and 32-ply laminates can be constructed with near complete isotropy while keeping the thickness to 480 and $960 \mu \mathrm{m}$, respectively. This would not be achievable with conventional composites.
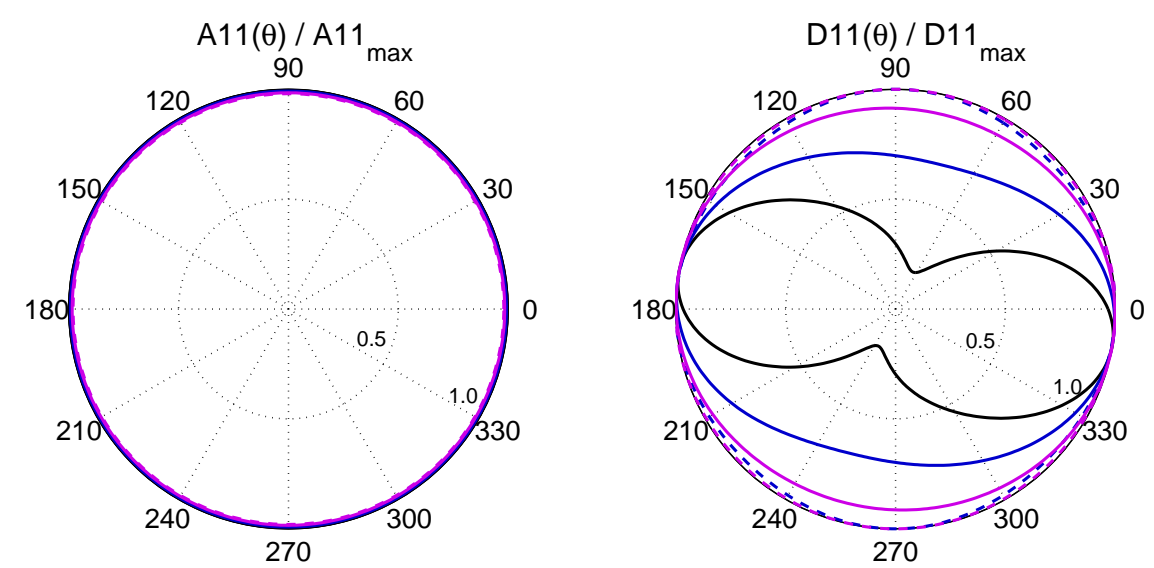

- Laminate 1 - Laminate $2 \mathrm{a}$ - - - Laminate $2 \mathrm{~b}$ - Laminate $3 \mathrm{a}$ - - - Laminate $3 \mathrm{~b}$

Figure 2. Normailized in-plane (A11) and bending (D11) stiffness at various orientations along the laminate.

Table 3. Reduction in laminate bending stiffness

\begin{tabular}{ccc}
\hline \hline Laminate ID & D11 $1_{\min } / \mathrm{D} 11_{\max }$ & Orientation $(\mathrm{deg})$ \\
\hline 1 & 0.186 & 65.0 \\
$2 \mathrm{a}$ & 0.687 & 77.5 \\
$2 \mathrm{~b}$ & 0.972 & 45.0 \\
$3 \mathrm{a}$ & 0.911 & 77.5 \\
$3 \mathrm{~b}$ & 0.993 & 45.0
\end{tabular}

To construct the mirror substrates, the laminates are assembled flat and then placed atop a mandrel used for cure. The mandrel is spherical with a $2.0 \mathrm{~m} \mathrm{ROC}$, thus producing nominally spherical mirror substrates. It is first treated with a release agent (Frekote $770 \mathrm{NC}$ ) in order to prevent the part from adhering to the surface. A 1/4" thick soft silicone pad is placed directly onto the backside of the laminate. This allows for an even pressure distribution to be obtained for subsequent vacuum-bagging and autoclave cure.

As the CFRP substrates contain no backing structure, it is a significant challenge to manufacture thin shells with highly-accurate shapes as large internal stresses can develop during the thermal processing. By using the balanced laminates listed in Table 2, and by subjecting the laminate to a uniform temperature distribution during cure, these stresses are predicted to balance out through the thickness of the laminate. However, factors such as variations in material properties, ply orientations, and temperature distributions across the part can cause imbalances to arise. These imbalanced stresses will warp the laminate upon cure and ultimately produce shape errors in the CFRP substrate. Initial studies showed that on average $50 \mu \mathrm{m}$ RMS of figure error, dominated by astigmatism, is present in $150 \mathrm{~mm}$ diameter CFRP substrates with a 16-ply laminate orientation.

Two methods to reduce these initial substrate errors have been implemented. First, a low-temperature, long duration cure profile was developed in order to reduce the magnitude of the thermal stresses during the 
cure. Laminates were cured at $80^{\circ} \mathrm{C}$ for $16 \mathrm{hrs}$, in comparison to the recommended $120^{\circ} \mathrm{C}$ cure provided by the manufacturer. Second, a deformable mandrel was constructed in order to null out systematic modes of initial figure error. By biasing the mandrel surface during the cure, a reduction in the observed magnitude of post-cure astigmatism was achieved. The combination of these two methods resulted in a $3 \times$ reduction in initial figure error for the 16-ply CFRP substrates at 16 - $17 \mu \mathrm{m}$ RMS. (See Steeves, J. $2015^{12}$ for a more detailed explanation of these two techniques).

\subsection{Nanolaminate Facesheet}

Surface roughness issues due to an effect known as "fiber print-through" are prevalent for CFRP-based optics. This occurs when the epoxy matrix contracts around the fibers during the curing process, causing them to protrude through the front surface of the mirror. Several methods to avoid this effect have been studied, however the use of a nanolaminate facesheet was implemented. Nanolaminates are multilayer metal foils formed by sputter deposition on a precision glass mandrel. ${ }^{13,14}$ During this process, the foil will replicated the surface of the mandrel, producing surfaces that are $<1 \mathrm{~nm}$ RMS in roughness. This foil is then transferred onto the front surface of the CFRP substrate using a room-temperature cure epoxy as shown in Figure 3. The liquid epoxy attenuates the fiber print-through by filling in the local roughness. Figure 4 compares the measured surface roughness of a bare CFRP substrate to one after integrating the nanolaminate facesheet. A surface roughness of $2.2 \mathrm{~nm} R$ a is achieved in comparison to the initial $49.5 \mathrm{~nm}$ Ra surface with fiber print-through. For the current effort $50 \mu \mathrm{m}$ thick nanolaminates composed of $\mathrm{Cu}$ and $\mathrm{Zr}$ layers were used. A terminal Au layer is implemented for the reflective front surface. Meter-class nanolaminates have been demonstrated in previous efforts and are thus scalable technologies.
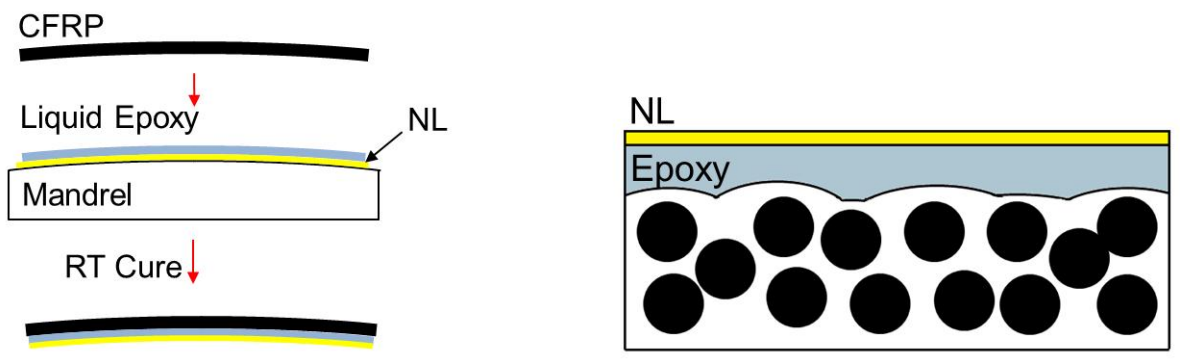

Figure 3. Schematic of nanolaminate bonding process used to mitigate fiber print-through.
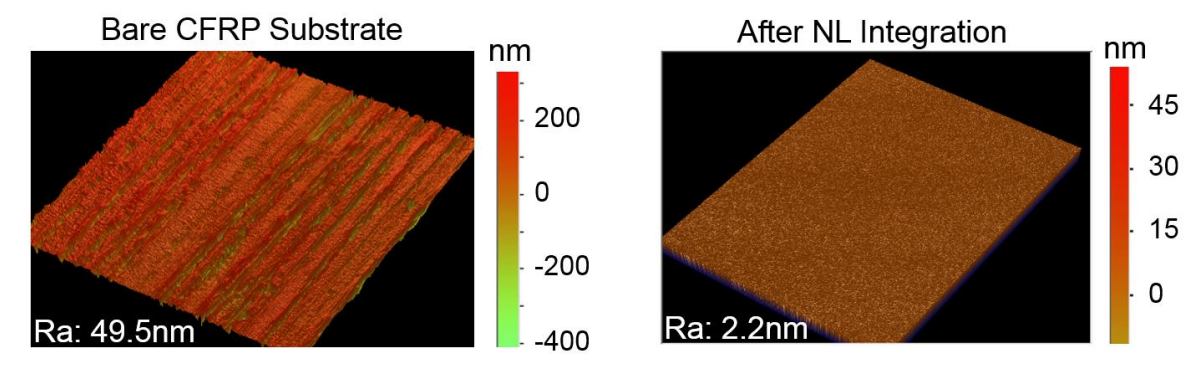

Figure 4. White Light Scanning Interferometer (Veeco Wyko) measurement of a) a bare CFRP substrate and b) a mirror prototype with a nanolaminate facesheet.

\subsection{Active Layer}

Several materials were considered for the active layer, however piezoelectric ceramics were chosen due to their high stiffness and actuation capabilities. Specifically, Lead-Zirconate-Titanate (PZT) was implemented for this study. The properties of this material can be found in Table 4. $125 \mu \mathrm{m}$ thick flat PZT plates were used 
as the active elements for the mirror substrate. The plates are initially $72.5 \times 72.5 \mathrm{~mm}$ square but trimmed to a section of an octagon using a high-speed cutting disk. The piezoelectric material is provided in a poled state with continuous nickel electrodes on either side. In order to apply a custom electrode pattern, these electrodes were removed using a wet nickel-stripping agent (Caswell B-9). A materials printer (Dimatix 2800) and silver nano-particle ink (Methode 9104) were then used to reprint the continuous ground plane on one side as well as the optimized pattern on the other (for information on the electrode pattern, see Laslandes, et. al. $\left.(2014)^{15}\right)$. These electrodes allow for an electric field to be applied through the thickness of the material, ultimately producing an in-plane strain in the material local to each electrode (i.e. d31-mode). The plates are bonded to the CFRP substrate using another room-temperature cure epoxy. Pressure is applied during this process using vacuum bagging techniques. Figure 5 displays the four PZT plates with the custom electrode pattern once bonded to the backside of the mirror.

Table 4. Material properties for PZT-5A.

\begin{tabular}{lr}
\hline \hline Property & Value \\
\hline Modulus, E (GPa) & 66.0 \\
Poisson's Ratio, $\nu$ & 0.35 \\
Piezoelectric Constant*, d31 (pC/N) & -375 \\
Maximum Electric Field*, $E_{\max }(\mathrm{MV} / \mathrm{m})$ & 0.8 \\
\hline *Measured value &
\end{tabular}

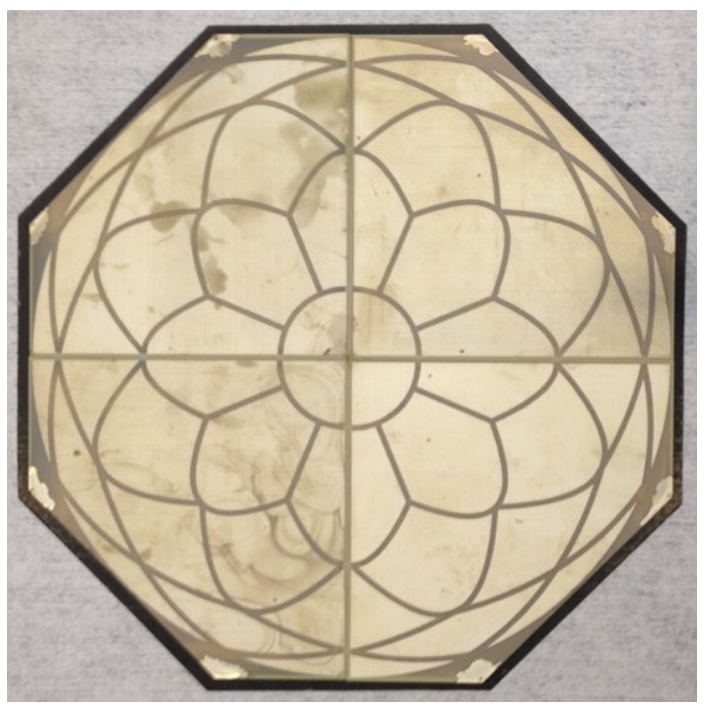

Figure 5. Backside of a $150 \mathrm{~mm}$ dia. active mirror prototype containing four PZT plates with a printed electrode pattern.

\subsection{Electrode Routing}

Bulky connecting wires are often required for active mirrors to address each of the independent actuation channels. These can potentially impart shape errors onto the mirror surface due to the stiffness of each wire. This is a particular problem for the current design as the mirrors are extremely thin and thus susceptible to even small mechanical constraints. To avoid this effect, conductive traces are printed on a $25 \mu \mathrm{m}$ thick Kapton routing layer. This routing layer is placed on the backside of the mirror and connections to the underlying electrodes are made using through-thickness vias and conductive epoxy. This allows the traces to be routed away from the active surface of the mirror and eventually to the downstream control electronics. The flexible Kapton layer allows the mirror to remain completely unconstrained during actuation. Figure 6 displays the backside of a mirror after integration of the electrode routing layer. 


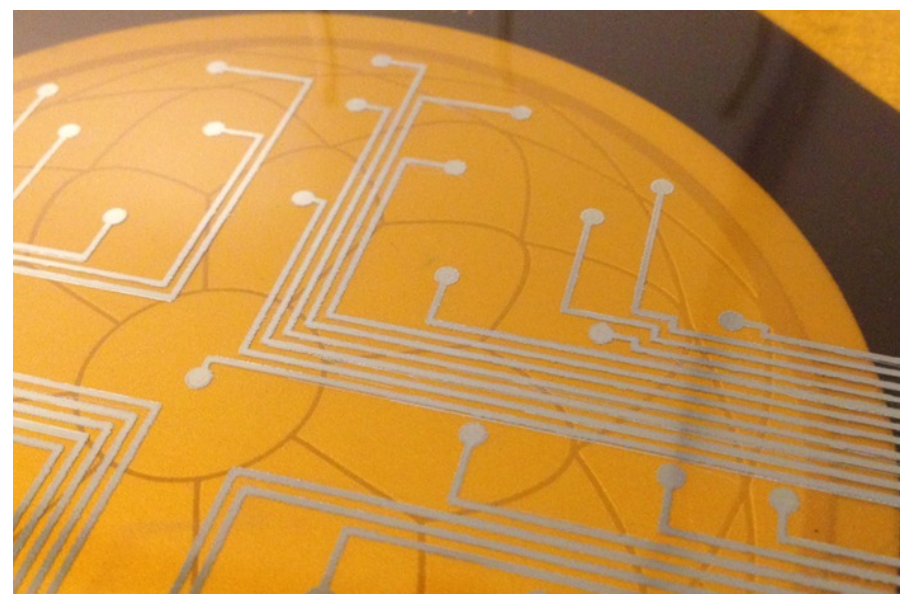

Figure 6. Backside of an active CFRP mirror after electrode routing layer integration.

\subsection{Fabrication Process}

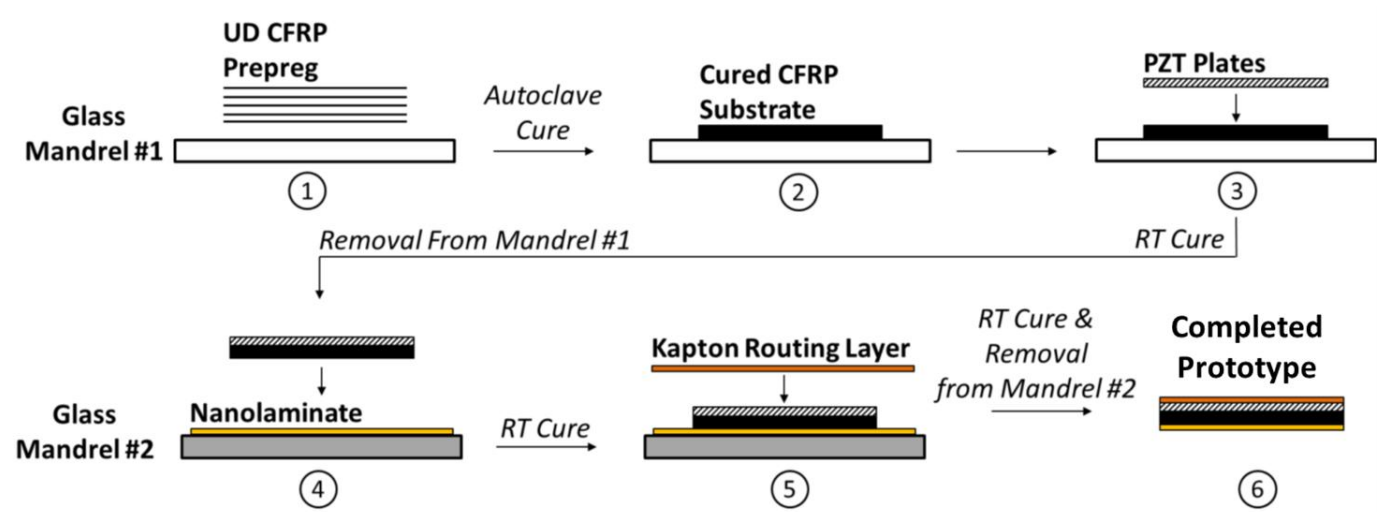

Figure 7. Fabrication process for the active mirror prototypes.

Figure 2.5 is a schematic of the overall fabrication process for the mirror prototypes. First, a lay-up of unidirectional carbon fiber prepreg is placed on top of a mandrel. The laminate is vacuum bagged and autoclave cured at elevated temperature and pressure to provide consolidation of the plies. During this process the CFRP material replicates the surface of the underlying mandrel, providing the overall figure of the mirror. Upon cure, the active layer layer is bonded to the backside of the CFRP substrate with the mandrel underneath to provide support. A common ground plane and patterned electrodes are printed on the bottom/top of the active layer before the bonding process takes place. Once cured, the assembly is removed from the underlying mandrel and bonded to a nanolaminate facesheet. The nanolaminate is deposited on a separate mandrel with the same figure as that used for the CFRP replication process, producing a matching part. Care must be taken in order to ensure a uniform thickness distribution of epoxy between the CFRP substrate and nanolaminate during this bonding process. The Kapton electrode routing layer is then incorporated onto the backside of the mirror using conductive epoxy, providing a method to route the actuation channels to the supporting electronics. Lastly, the completed active mirror is parted from the secondary mandrel. Figure 8 displays the front and back surface of a fully assembled $150 \mathrm{~mm}$ dia. prototype.

\section{SHAPE CORRECTION PREDICTIONS}

The actuation capabilities of the mirrors were modeled using Abaqus CAE/Standard 6.12 using conventional elastic shell elements with reduced integration (S4R). In the model a thermal expansion analog was used 


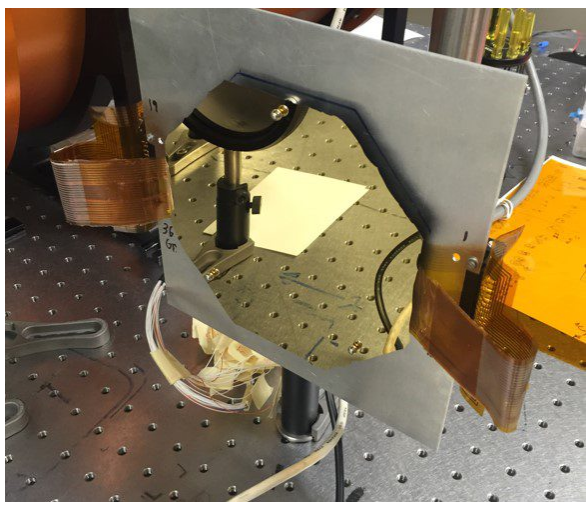

(a)

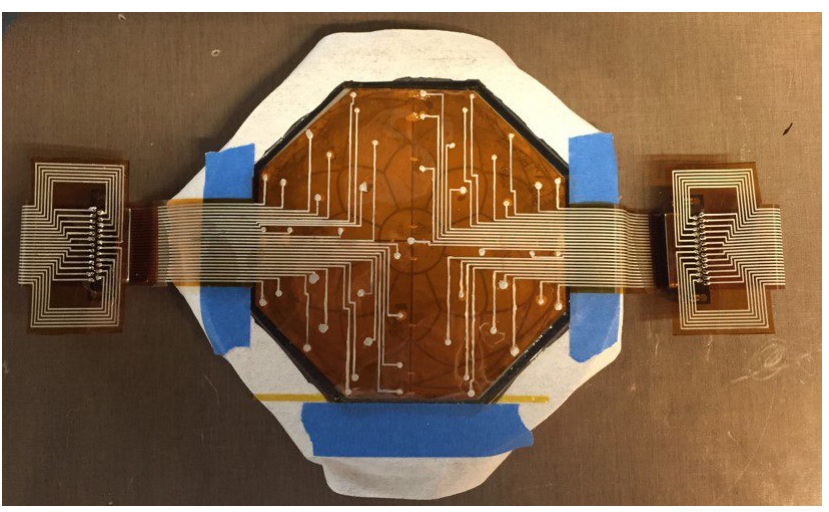

(b)

Figure 8. a) Front and b) back surfaces of $150 \mathrm{~mm}$ diameter prototypes.

where the linear coefficient of thermal expansion and a subsequent temperature change was used to mimic the linear piezoelectric response and applied electric field. Through a custom Python script, the influence function of each actuator was determined by defining a non-zero expansion value for the actuator of interest. The expansion value was scaled such that a unit temperature change corresponded to the application of 1 $\mathrm{V}$. The model assumed that there were no gaps between adjacent actuators, however it has been determined that this is of significant importance and should thus be studied in detail.

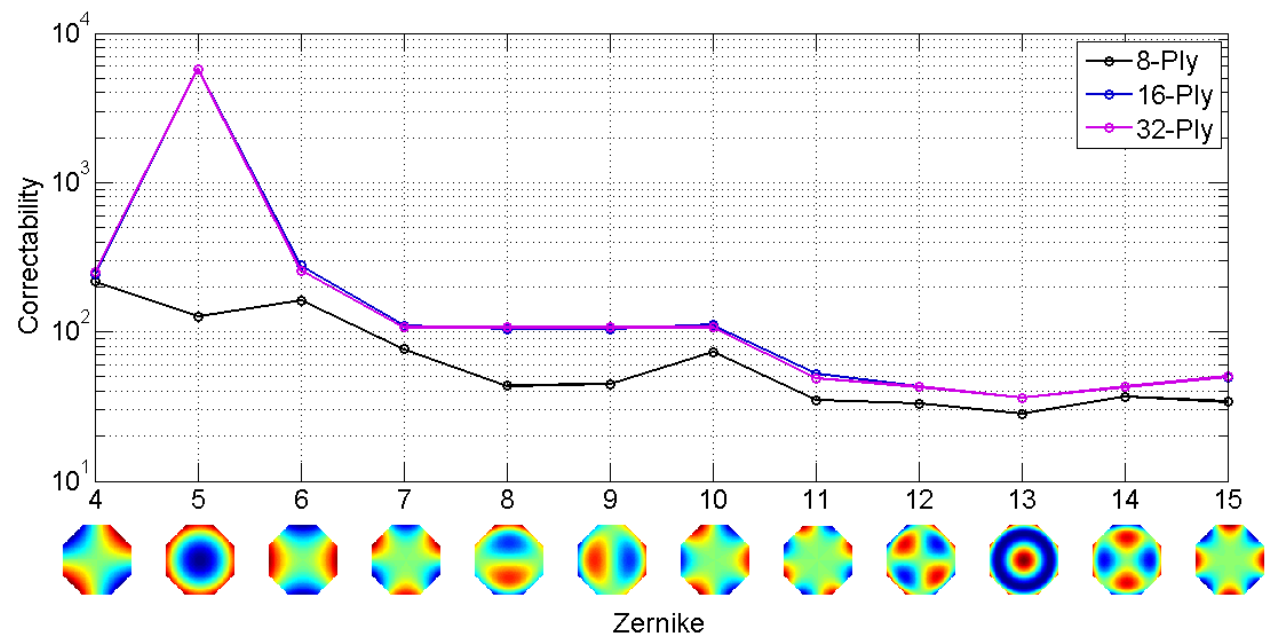

Figure 9. Correctability of Zernike modes for 8, 16 and 32-ply mirror designs.

Figure 9 displays the correctability of the three mirror designs over various Zernike modes. Correctability is defined as the ratio of residual error to initial error for each specific mode before actuator saturation occurs:

$$
\text { Correctability }_{k}=\frac{\left(Z_{k}^{z e r n}\right)_{r m s}}{\left(r_{k}^{z e r n}\right)_{r m s}}
$$

where $Z_{k}^{z e r n}$ is the $\mathrm{k}^{t h}$ Zernike mode and $r_{k}^{z e r n}$ is the residual shape error after correction of that mode. It should be noted that Zernike modes are only valid over a circular domain. To accommodate the octagonal shape of the mirror designs, a circular domain encompassing the entire mirror substrate was defined and then truncated to the octagonal form-factor. 
Several observations can be made from Figure 9. First, the performance of the 16 and 32-ply designs is near identical over all Zernike modes. This is due to the similar laminate orientation producing near isotropic substrates. As previously stated, the 8-ply laminate is not isotropic in bending and therefore will not have the same behavior as the other two designs. A decrease in correctability is observed over all modes for this laminate. Second, for the 16 and 32-ply laminates the correctability is highest for the change in bulk curvature, often denoted as defocus (mode 5). This mode is achieved by uniformly actuating the entire active layer at near equal voltages. Therefore, shape errors due to differing voltage levels at neighboring actuators do not arise. However, for the 8-ply design the vast difference in bending stiffness in orthogonal directions does not allow for equal curvature changes upon uniform actuation. Therefore, the correctability in defocus is significantly reduced. It is also observed that all mirror designs possess relatively high levels of correctability over the two astigmatic modes (mode 4 and 6). This is due to the chosen electrode pattern as it was optimized for astigmatism-based deformations. Finally, correctability is observed to decrease for increasing Zernike modes as the finite size of each electrode limits the ability to correct for higher spatial-frequency errors.

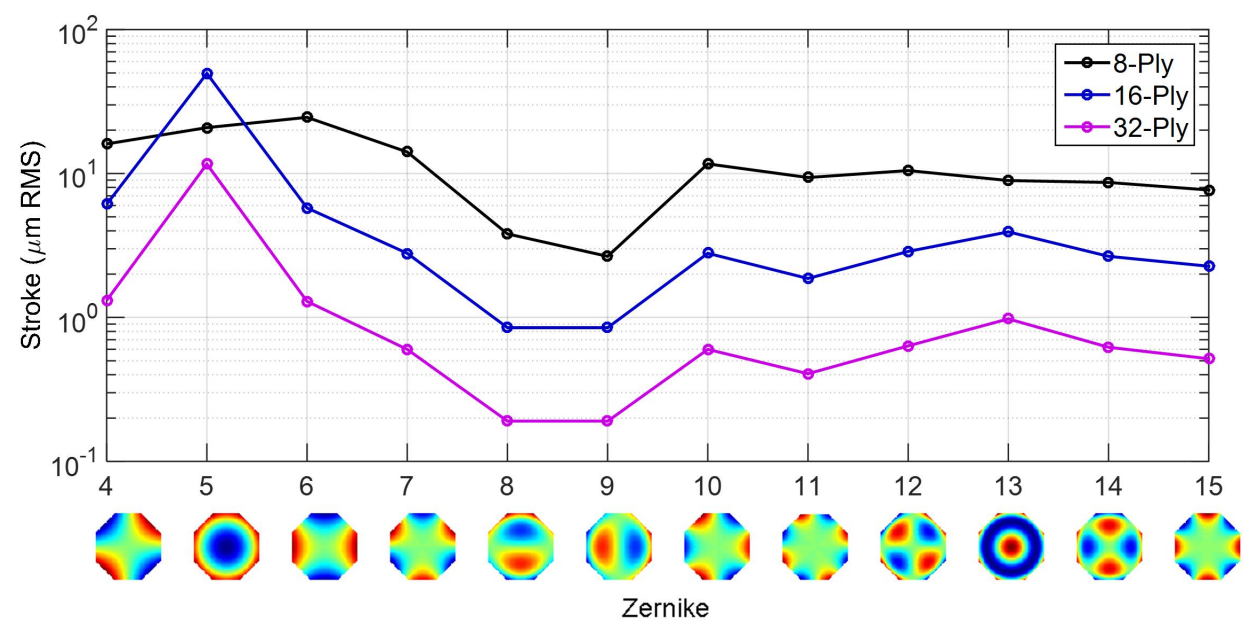

Figure 10. Zernike stroke for 8, 16 and 32-ply mirror designs.

The stroke of the mirror for each Zernike mode is presented in Figure 10. Stroke is defined as the maximum achievable RMS Zernike amplitude before saturation of the first actuator:

$$
\begin{array}{r}
\text { Stroke }_{k}=\left(Z_{k}^{z e r n}\right)_{r m s} \\
\text { s.t. }\left|V_{i}\right|_{\max }=100 \mathrm{~V} .
\end{array}
$$

For all modes other than defocus, stroke decreases as the substrate ply-count increases due to the increased bending stiffness. The defocus mode for the 8-ply substrate is decreased which is again attributed to its non-isotropic properties.

\section{REVERSE HARTMANN METROLOGY}

Traditional optical measurement techniques were unable to capture 1) the initial figure and 2) the large actuation capabilities associated with mirrors of this design. Therefore, a custom metrology system was developed using a Reverse Hartmann test. To understand the Reverse Hartmann test, it is helpful to briefly review the classic Hartmann test. ${ }^{16}$ A full description can be found in the classic book on optical shop testing by Malacara. ${ }^{17}$ This test has a long history, and has found broad applicability because: 1) it is simple to implement, 2) easy to interpret and 3) has large dynamic range. To begin, a "mask" is placed over the input 
pupil of the optical system. The mask is composed of a fixed set of clear apertures in an otherwise opaque screen. When illuminated, the holes generate a set of beams which behave as geometric rays. These rays traverse the complete optical system until their locations are measured just before an internal focus - ideally the final focus. The deviation of these rays from a scaled version of the input pattern is a measure of the optical quality of the system. Quantitatively, imagine the Hartmann mask sharply reimaged at the output pupil of the system. The regular ray pattern of the input mask will be reformed (absent any distortion in the imaging between these two planes). However, the output pupil will have a phase error due to the imperfections in the system. At each ray in the output pupil, the phase gradient will cause the ray to deviate from what would be its unaberrated path. The magnitude of the ray displacement is simply given by the slope of the rays (i.e. phase gradient) multiplied by the distance the ray traverses as measured from the output pupil, as follows:

$$
\delta x=\frac{\delta \phi}{\delta x} l,
$$

and

$$
\delta y=\frac{\delta \phi}{\delta y} l,
$$

where $\phi$ is the phase in the output pupil, and $l$ is the distance to the output pupil. This measurement can also be inverted to produce a quantified phase map in the output pupil. This can be done in one of two ways: 1) modeling of the optical system to determine the spot motions for a given aberration, or 2) measurement of the spots for a given disturbance to the system.

The Reverse Hartmann test: 1) swaps the source and test planes, and 2) reverses the beam propagation direction. From a point source conjugate to the final focal plane of the system, we send a perfectly diverging beam (from a single-mode fiber) back towards the entrance aperture of the system. This beam passes through a mask which is a grid of regularly spaced holes, illuminating the mirror with a uniform grid of spots. This grid is then reflected off of the mirror and propagated to a flat projection screen under nominally collimated conditions. A camera captures an image of the grid pattern on the screen. Spot centroiding algorithms are used to detect distortions in this pattern, corresponding to phase errors in the mirror under test. Figure 4 displays the lab implementation of the Reverse Hartmann testbed.

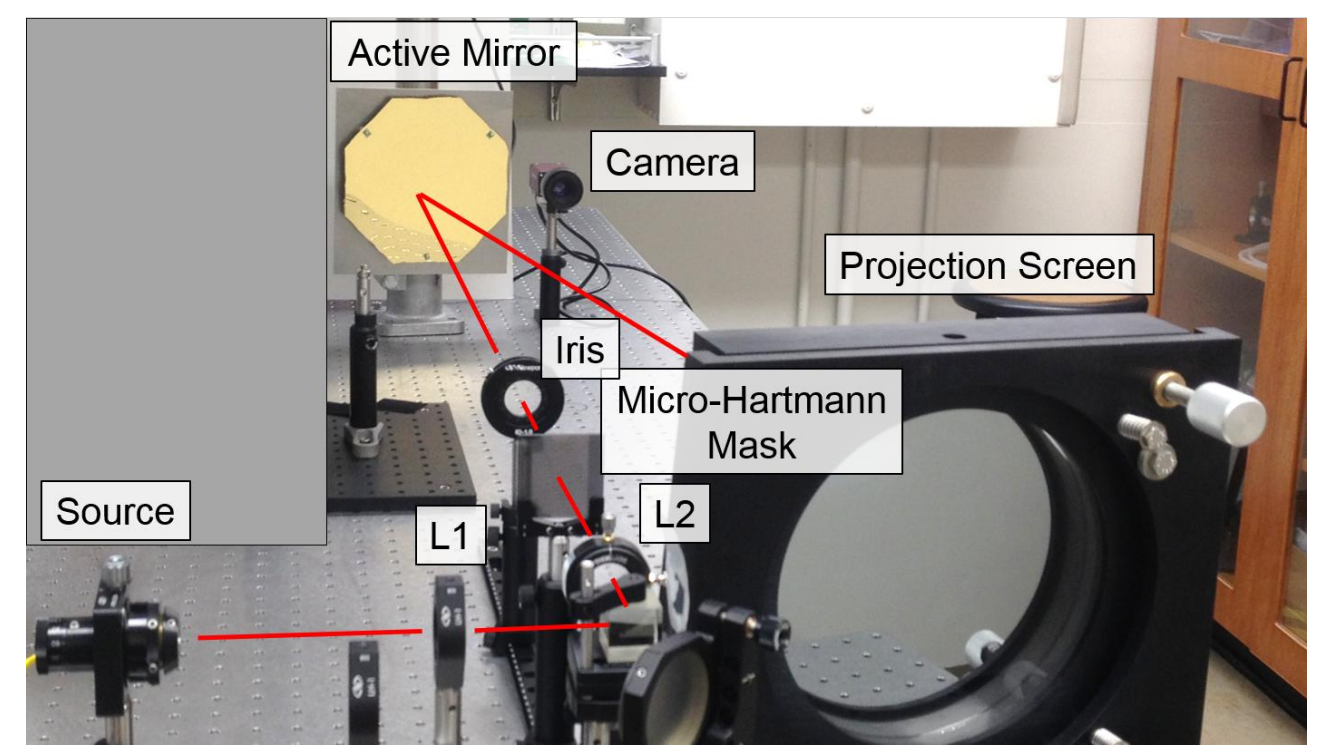

Figure 11. Reverse Hartmann testbed used for mirror characterization. 

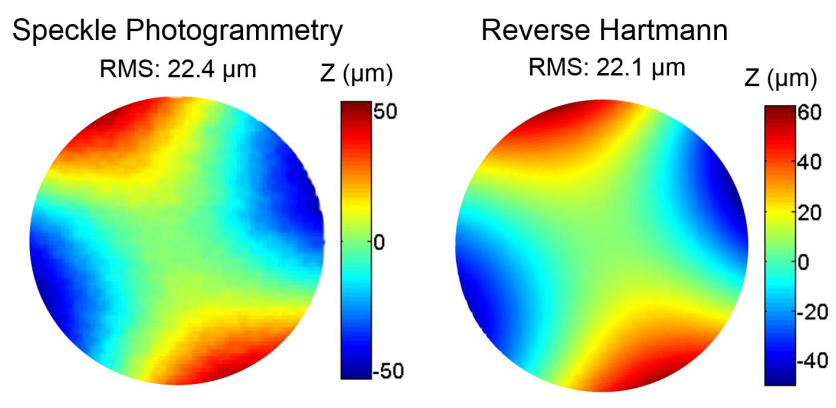

(a)
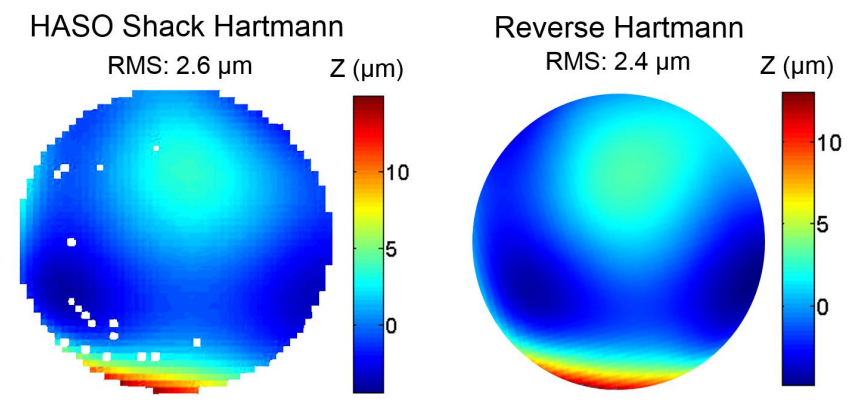

(b)
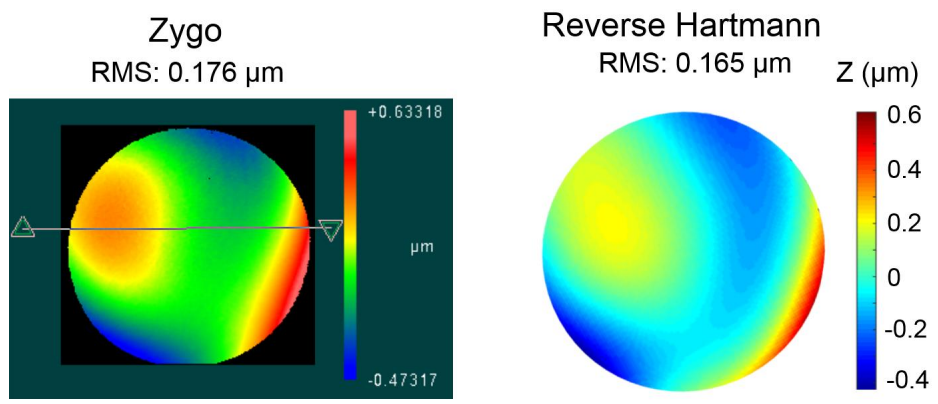

(c)

Figure 12. Comparison of Reverse Hartmann measurements to measurements made through a) speckle photogrammetry, b) a Shack-Hartmann wavefront sensor, and c) a Zygo interferometer.

In order to validate the Reverse Hartmann testbed, as well as demonstrate the dynamic range of the system, several measurements were made on test optics with varying surface quality and phase errors. These measurements were then repeated using the same test optics but using systems with calibrated phase estimates. Figure 12 displays these measurement comparisons. The first compares measurements obtained from a speckle photogrammetry system (Correlated Solutions, Vic3D ${ }^{18}$ ) on a bare CFRP substrate subsequent to autoclave curing. The sample was uncoated and without a nanolaminate facesheet, and thus had a relatively low reflectance front surface. As the shown in the measurements, a large amplitude astigmatic phase error was present in the substrate with an RMS value of approximately $22 \mu \mathrm{m}$. The Reverse Hartmann testbed was able to accurately capture the figure of this substrate with good agreement to the photogrammetry system despite the large amplitude error and poor surface quality. The second measurement, shown in Figure 12(b), was a measurement of a CFRP substrate after bonding a $50 \mu \mathrm{m}$ thick nanolaminate facesheet to its front surface. This significantly increased the surface quality of the substrate. Using a traditional Shack-Hartmann (HASO $76^{19}$ ) the figure of this mirror was shown to contain significant mid-spatial frequency errors at an amplitude of approximately $2.5 \mu \mathrm{m}$ RMS. It can be seen that the Reverse Hartmann was also able to cap- 
ture the mode and magnitude of these errors. Finally a rigid glass mirror, manufactured with an off-axis hyperboloid prescription, was tested with a Zygo interferometer. The Reverse Hartmann test was able to accurately capture the low amplitude errors of this optic ( $\sim 170 \mathrm{~nm}$ RMS $)$. The results of these tests clearly demonstrate the versatility of this technique to characterize optical figure errors of varying magnitude.

\section{MIRROR PERFORMANCE}

\subsection{Figure Correction}

The initial resting figure of the mirror, shown in Figure 13(a), is $6.25 \mu \mathrm{m}$ RMS and is dominated by astigmatism due to stresses which develop during the manufacturing process. A constrained linear least squares fitting algorithm is used with measurements from the Reverse Hartmann apparatus to flatten the mirror in closed-loop. The best flat achieved was $0.206 \mu \mathrm{m}$ RMS over $80 \%$ of the mirror surface and the resulting residual is shown in Figure 13(b). The voltage commands used to achieve the best flat are shown in Figure 13(c); several edge actuators have saturated, however the central actuators are well below the voltage limits. This is a significant improvement over previous versions of the mirror ${ }^{20,21}$ whose figure correction was voltage limited due to initial shape errors.

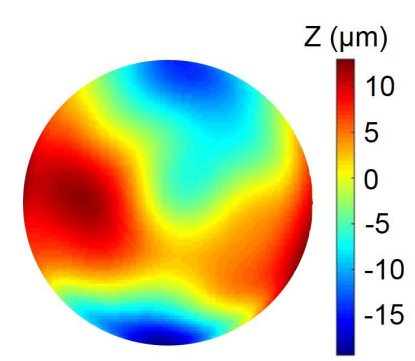

(a)

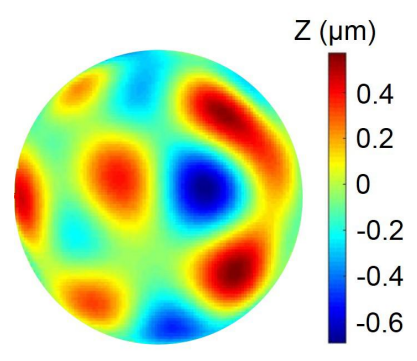

(b)

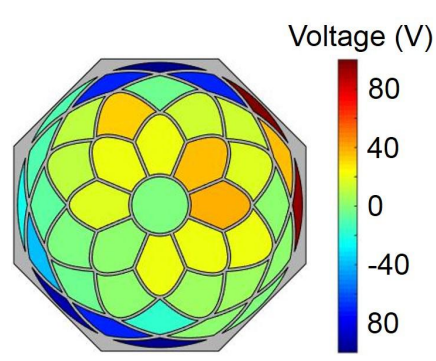

(c)

Figure 13. (a) Initial resting shape: $6.25 \mu \mathrm{m}$ RMS, $32.4 \mu \mathrm{m}$ PV. (b) Best flat surface error after closed loop correction: $0.206 \mu \mathrm{m}$ RMS, $1.26 \mu \mathrm{m}$ PV. (c) Map of final actuator voltage commands between $\pm 100 \mathrm{~V}$.

It should be noted the the $\lambda / 3$ surface was achieved with only 39 active actuators, as two actuators were shorted due to wiring problems. Further, the residual error is given for spatial frequencies within the bandwidth of the mirror. Approximately $120 \mathrm{~nm}$ of RMS surface error exists in higher order modes, and further study into actuator density and pattern optimization as well as manufacturing processes is on-going in order to address the overall surface error.

\subsection{Differential Mode Shapes}

To establish the ability of the mirror and actuator pattern to take on low order modes using the full actuation range, a differential modal control loop was implemented. This consisted of measuring the resting shape of the mirror and then driving it in closed loop to add a selected low order Zernike mode to its resting shape. A command starting point was computed by projecting the selected Zernike mode onto the measured influence functions. Examples of the results are given for astigmatism and coma in Figures 14 and 15 which show the target shape (left), the theoretical best fit residual error (middle), and the measured residual error after closed loop control (right). In both examples, the RMS of the measured residual is within a few nanometers of the predicted fit, however there are deviations in the pattern of the residuals which lead to an investigation into the linearity of the influence functions. 


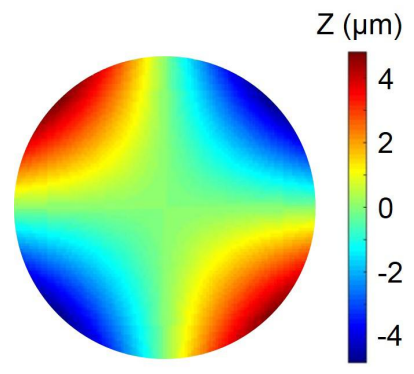

(a)

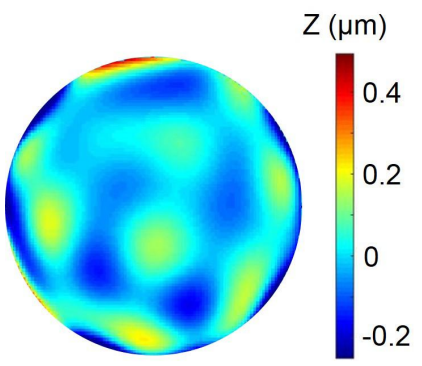

(b)

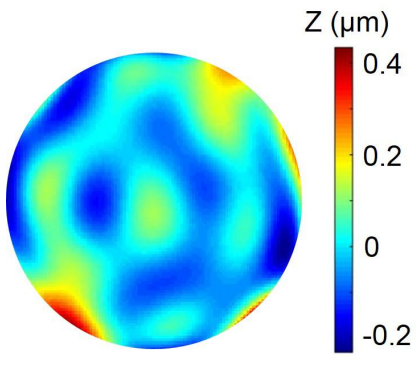

(c)

Figure 14. (a) Target shape: Astigmatism $2.0 \mu \mathrm{m}$ RMS. (b) Theoretical residual fitting error using measured influence functions: $88.0 \mathrm{~nm}$ RMS. (c) Measured residual after closed loop differential modal control: $91.6 \mathrm{~nm}$ RMS.

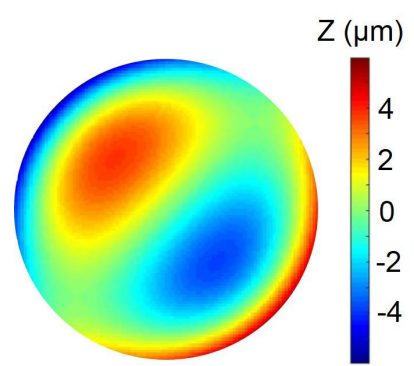

(a)

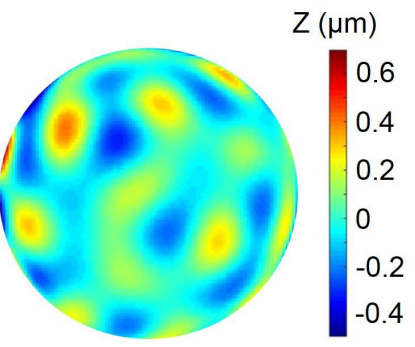

(b)

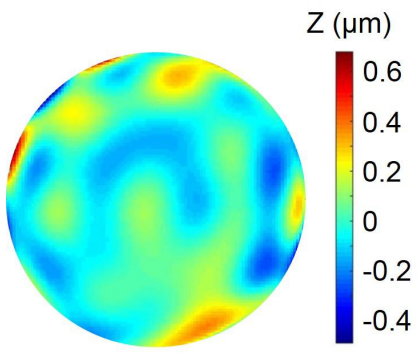

(c)

Figure 15. (a) Target shape: Coma $2.0 \mu \mathrm{m}$ RMS. (b) Theoretical residual fitting error using measured influence functions: $119 \mathrm{~nm}$ RMS. (c) Measured residual after closed loop differential modal control: $123 \mathrm{~nm}$ RMS.

\subsection{Actuator Coupling}

Due to some noted discrepancies between the theoretical projection of system performance based on measured influence functions, it was postulated that some non-linear effects may be impacting the mirror performance. To determine the first order magnitude of this effect, the influence functions of several combinations of adjacent actuators were co-added assuming linear influence. These actuator pairs were then actuated simultaneously by applying equal voltage to their electrodes, and their response was again measured. The difference between the linear combination of individual influences and the combined influences was found to be consistent in magnitude and shape with the pattern shown in Figure 16. Not all adjacent actuator combinations have been analyzed, and neither have non-adjacent actuator combinations. While the specific source of this effect has not yet been investigated, it is believed that this could be due to stress dependent changes in the material properties of the actuator material, and/or non-linear electric field effects around the edges of each electrode. 


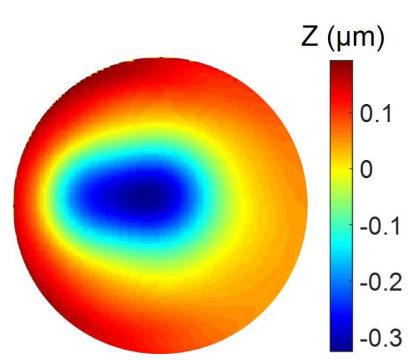

(a)

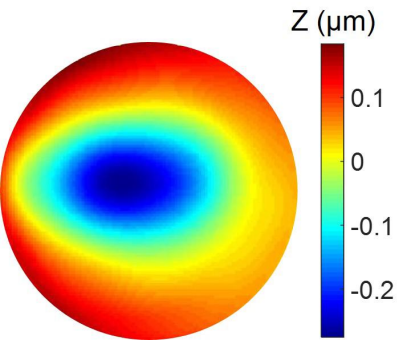

(b)

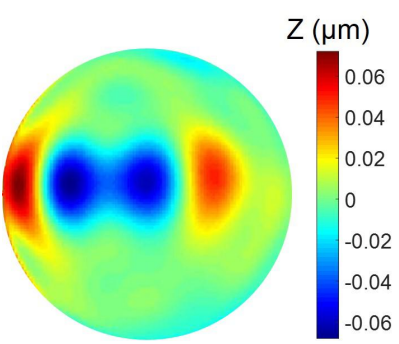

(c)

Figure 16. Actuator coupling: (a) The linear sum of Actuator 1 and Actuator 8 influence functions. (b) Simultaneous actuation of Actuators 1 and 8. (c) Difference between (a) and (b).

\section{CONCLUSIONS}

A novel active mirror technology based on carbon fiber composites and replication techniques has been developed. Additional material layers are implemented into the mirror structure in order to provide the reflective surface, actuation capabilities, and electrode routing. Mirrors of this design are thin $(<1.0 \mathrm{~mm})$, lightweight $\left(\sim 2.5 \mathrm{~kg} / \mathrm{m}^{2}\right)$, and exhibit large integrated actuation capabilities. Finite element simulations show high correctability and actuation stroke over low-order Zernike modes with only 42 independent actuators. A custom metrology testbed was developed based on a Reverse Hartmann test in order to characterize the mirror surface. The system was demonstrated to have a large dynamic range with the ability to capture $>100 \mu \mathrm{m}$ PV figure errors down to sub-micron accuracy. This system was then used to characterize the performance of a $150 \mathrm{~mm}$ dia. active mirror prototype. Figure correction experiments were performed, demonstrating the ability to correct $6.25 \mu \mathrm{m}$ RMS initial error to $205.5 \mathrm{~nm}$ RMS, with two dead actuators limiting the performance.

\section{ACKNOWLEDGMENTS}

A part of this research was carried out at the Jet Propulsion Laboratory, California Institute of Technology under a contract with the National Aeronautics and Space Administration (NASA).

\section{REFERENCES}

1. J. Dalcanton, S. Seager, S. Aigrain, S. Battel, N. Brandt, C. Conroy, L. Feinberg, S. Gezari, O. Guyon, W. Harris, et al., "From cosmic birth to living earths: the future of uvoir space astronomy," arXiv preprint arXiv:1507.04779, 2015.

2. M. R. Bolcar, K. Balasubramanian, M. Clampin, J. Crooke, L. Feinberg, M. Postman, M. Quijada, B. Rauscher, D. Redding, N. Rioux, et al., "Technology development for the advanced technology large aperture space telescope (atlast) as a candidate large uv-optical-infrared (luvoir) surveyor," in SPIE Optical Engineering + Applications, pp. 960209-960209, International Society for Optics and Photonics, 2015.

3. C. Baffes, T. Mast, J. Nelson, E. Ponslet, V. Stephens, L. Stepp, and E. C. Williams, "Primary mirror segmentation studies for the thirty meter telescope," in SPIE Astronomical Telescopes+ Instrumentation, pp. 70180S-70180S, International Society for Optics and Photonics, 2008.

4. P. C. Chen, T. T. Saha, A. M. Smith, and R. Romeo, "Progress in very lightweight optics using graphite fiber composite materials," Optical Engineering, vol. 37, no. 2, pp. 666-676, 1998.

5. P. C. Chen, C. W. Bowers, M. Marzouk, and R. C. Romeo, "Advances in very lightweight composite mirror technology," Optical Engineering, vol. 39, no. 9, pp. 2320-2329, 2000.

6. J. D. Hochhalter, Replicated mirrors using carbon fiber reinforced polymers. PhD thesis, University of New Mexico, 2005. 
7. J. Massarello, B. deBlonk, and A. Maji, "Rapid fabrication of mirrors with nanolaminate facesheets and composite structures," in International Congress and Exposition on Experimental and Applied Mechanics, Society for Experimental Mechanics, 2004.

8. S. Kendrew, P. Doel, D. Brooks, C. Dorn, C. Yates, R. M. Dwan, I. Richardson, and G. Evans, "Development of a carbon fiber composite active mirror: design and testing," Optical Engineering, vol. 45, no. 3, pp. 033401-033401, 2006.

9. K. Patterson and S. Pellegrino, "Ultralightweight deformable mirrors," Applied optics, vol. 52, no. 22, pp. 5327-5341, 2013.

10. K. Patterson, S. Pellegrino, and J. Breckinridge, "Shape correction of thin mirrors in a recongurable modular space telescope," in SPIE Astronomical Telescopes + Instrumentation, pp. 773121-773121, International Society for Optics and Photonics, 2010.

11. R. M. Jones, Mechanics of composite materials. CRC Press, 1998.

12. J. Steeves, Multilayer Active Shell Mirrors. 2015.

13. T. Barbee, "Nanolaminate thin-shell mirror structures," in Society of Photo-Optical Instrumentation Engineers (SPIE) Conference Series, vol. 5166, 2003.

14. G. S. Hickey, S.-S. Lih, and T. W. Barbee Jr, "Development of nanolaminate thin-shell mirrors," in Astronomical Telescopes and Instrumentation, pp. 63-76, International Society for Optics and Photonics, 2002.

15. M. Laslandes, S. Pellegrino, J. Steeves, and K. Patterson, "Optimization of electrode configuration in surface-parallel actuated deformable mirrors," in SPIE Astronomical Telescopes + Instrumentation, pp. 914843-914843, International Society for Optics and Photonics, 2014.

16. J. Hartmann, Bemerkungen über den Bau und die Justirung von Spektrographen. Springer, 1900.

17. D. Malacara, Optical shop testing, vol. 59. John Wiley \& Sons, 2007.

18. "Correlated solutions." http://www.correlatedsolutions.com/, 2016.

19. "Imagine optic." http://www.imagine-optic.com/, 2016.

20. J. Steeves, M. Laslandes, S. Pellegrino, D. Redding, S. C. Bradford, J. K. Wallace, and T. Barbee, "Design, fabrication and testing of active carbon shell mirrors for space telescope applications," in SPIE Astronomical Telescopes+ Instrumentation, pp. 915105-915105, International Society for Optics and Photonics, 2014.

21. J. Steeves and S. Pellegrino, "Ultra-thin highly deformable composite mirrors," in 54th AIAA Structures, Structural Dynamics, and Materials Conference, 2013. 